\title{
QUANTUM GENERATION AND DETECTION OF INCOHERENT PHONONS IN SUPERCONDUCTORS
}

\author{
W. Eisenmenger* and A. H. Dayem \\ Bell Telephone Laboratories, Murray Hill, New Jersey
}

(Received 7 December 1966)

The energy gaps in the quasiparticle excitation spectrum of superconductors cover a corresponding frequency range from about 1 to $1000 \mathrm{GHz}$. The relaxation and recombination of excited quasiparticles occur mostly via electron-phonon interaction. ${ }^{1,2}$ Thus a superconductor may be utilized as a quantum phonon generator and detector in a frequency range where conventional means of generation become prohibitively difficult. We report on experiments designed to investigate the possibility of using superconducting tunnel diodes for phonon generation and detection.

The phonon generator is a $\mathrm{Sn}-\mathrm{SnO}_{2}-\mathrm{Sn}$ tunnel diode evaporated on an optically flat surface of a 1-cm-long sapphire single crystal. The receiver is a similar diode on the opposite face of the crystal. The generating diode is biased at a voltage $V_{1} \geqslant 2 \Delta$, while the receiver is at $V_{2}<2 \Delta$, where $2 \Delta$ is the energy gap in electron volts. Injection via tunneling provided the excited quasiparticles in both films constituting the generator. Some of the phonons emitted in the relaxation processes propagate in the sapphire in rectilinear trajectories with little attenuation. ${ }^{3}$ The phonons incident on the receiving diode produce a change in the tunneling current which, as shown below, depends on both the frequency and number of the phonons. Time-of-flight pulse technique is used to separate in time the generation and detection phenomena.

Our major findings are the following: (1) An excited quasiparticle of energy $E$ (measured from the Fermi level) relaxes first to the top of the energy gap emitting a phonon of energy $E-\Delta$. This is followed by a recombination of pairs of quasiparticles of opposite spins emitting phonons of energy $2 \Delta$. (2) Only $\sim 80 \%$ of the recombination occurs via emission of phonon of energy $2 \Delta$. (3) The dominant mechanism in the receiving process is the dissociation of a Cooper pair due to absorption of a phonon of energy $\geqslant 2 \Delta$. (4) The over-all quantum efficiency, defined by $2 \pi r^{2} I_{2}{ }^{\prime} / A I_{1}$ is typically of the order of $1 \%$. Here $I_{1}$ is the tunnel current in the generator, $I_{2}{ }^{\prime}$ is the current increment in the receiver due to phonon interaction, $A$ is the area of the tunnel diode, and $r$ is the distance between the two diodes.

The tunnel diodes were prepared in the form of crossed stripes of tin of about $1 \mathrm{~mm}$ width. The thickness is $\sim 1000 \AA$ for the stripe adjoining the sapphire and $\sim 3000 \AA$ for the superimposed stripe. The normal-state resistance of the junctions tested ranged between 0.01 and $0.3 \Omega$ at $1.2^{\circ} \mathrm{K}$. The sapphire single crystal is of a high quality synthetic material with a low dislocation density of $200 \mathrm{~cm}^{-2}$. It is cylindrical in shape with a diameter of 0.95 $\mathrm{cm}$ and length $1.04 \mathrm{~cm}$. The cylindrical axis is parallel to the crystallographic $a$ axis to within $\pm 1^{\circ}$. The polished surfaces are plane and parallel to within $1 / 20$ of the sodium light wavelength. The experiments were carried out with the sapphire (and the diodes) in direct contact with the helium bath. Each diode has four terminals enabling the usual dc measurements as well as pulsed operation. We used dc pulses of repetition rate $10 \mathrm{kHz}$ and duration from 0.2 to $5 \mu \mathrm{sec}$. To suppress the dc Josephson current it was found necessary to apply an external magnetic field of $\sim 10 \mathrm{G}$ parallel to the diode plane.

The experimental results are summarized in Figs. 1, 2, and 3. Current pulses are applied to the generating diode, and a constant dc bias of $0.8 \mathrm{mV}$ is applied to the receiving diode. The pulse output voltage of the receiver was fed through a pulse amplifier into a sam-

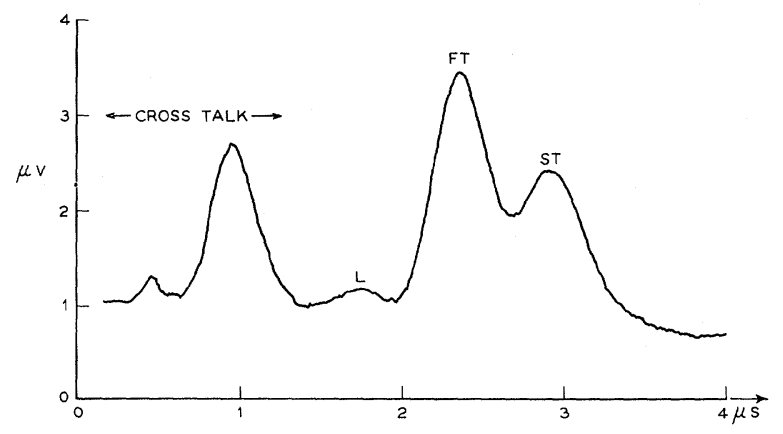

FIG. 1. Plot of received signal versus time. The three peaks $L, F T$, and $S T$ correspond, respectively, to the longitudinal and the fast and slow transverse modes of propagation in sapphire. 
pling scope, the output of which was used to drive an $X-Y$ recorder. Figure 1 shows a plot of the receiver signal versus time. After the peaks at the left, which result from crossstalk between generator and receiver circuits, one observes three peaks corresponding to the longitudinal and the fast and slow transverse modes of propagation in the sapphire. Because of our uncertainty concerning the over-all delay in the circuits used, we cannot estimate the absolute velocity of the various modes in sapphire using the results shown in Fig. 1. Previous ly published data ${ }^{4}$ on the phase velocities in the $a$ direction of sapphire are $11.09 \times 10^{5} \mathrm{~cm}$ $\mathrm{sec}^{-1}$ for the longitudinal mode and $6.693 \times 10^{5}$ and $5.694 \times 10^{5} \mathrm{~cm} \mathrm{sec}^{-1}$ for the fast and slow transverse modes, respectively. Using these values and the length of our sapphire crystal, we calculate a delay of $0.618 \mu \mathrm{sec}$ for the fast and $0.890 \mu \mathrm{sec}$ for the slow transverse waves, both referred to the arrival time of the longitudinal mode. The corresponding values estimated from Fig. 1 are 0.610 and $1.15 \mu \mathrm{sec}$, respectively. The agreement for the fast transverse mode is good and indicates that the received signal is due to phonons propagating in the sapphire from one end to the other. The disagreement for the slow transverse mode is rather large. It cannot be attributed to dispersion since the phonon frequency is $\sim 300$ $\mathrm{GHz}$, too small for dispersion in sapphire to set in. It is quite possible that diffuse reflection at the cylindrical walls contributes to the apparent delay of the slow transverse mode.

Figure 2 shows the relationship between the pulse amplitudes of the received ourput voltage $V_{2}{ }^{\prime}$ and the generator input current $I_{1}$. The receiver dc bias is again kept constant at $0.8 \mathrm{mV}$ while the sampling point is now fixed at the peak of the fast transverse mode. Also shown in Fig. 2 is the $I-V$ characteristic of the generating diode measured under pulsed operation. Corresponding voltages in units of gap energy are inserted. One observes that the received phonon signal increases almost linearly with current as long as the generating diode voltage $V_{1}$ is smaller than $4 \Delta$. At $V_{1}=4 \Delta$ the slope changes sharply by a factor of about 2.5 but the increase remains linear up to $V_{1}=6 \Delta$. From there on the dependence is nonlinear. This behavior is more clearly shown in Fig. 3, which represents the measured derivative of Fig. 2. The derivative has been obtained experimentally by applying to the gen-

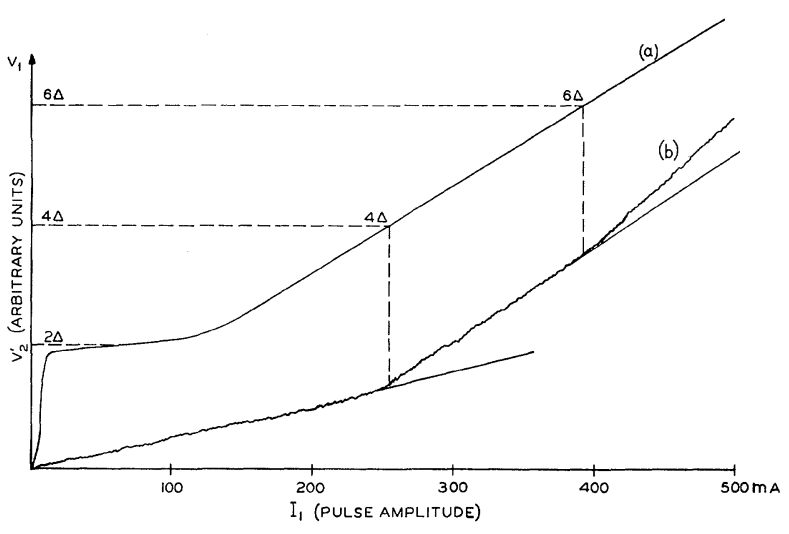

FIG. 2. (a) $I-V$ characteristic of the generating diode measured under pulsed conditions with voltage $V_{1}$ expressed in units of $2 \Delta$. (b) Relationship between the pulse amplitudes of the receiver output voltage $V_{2}^{\prime}$ and the generator input current $I_{1}$. The receiver dc bias is at $0.8 \mathrm{mV}$ and the sampling point is fixed at the peak of fast transverse mode.

erating diode a fixed small current pulse superimposed on a variable dc bias. The receiver pulse signal is then plotted versus the generator dc bias current as shown in Fig. 3. The sharp step occurring at $V_{1}=4 \Delta$ is clearly preceded and followed by regions of nearly constant values. There is probably some additional structure at $V_{1}=2 \Delta$. Also, the dependence of the received signal on the receiver bias $V_{2}$ shows many interesting features, especially for $V_{2}<\Delta$, which probably result from some interaction with the Josephson effect. This, however, will not be pursued any further in this publication, awaiting additional experimental data.

The observations depicted in Figs. 2 and 3 indicate the predominance of quantum process-

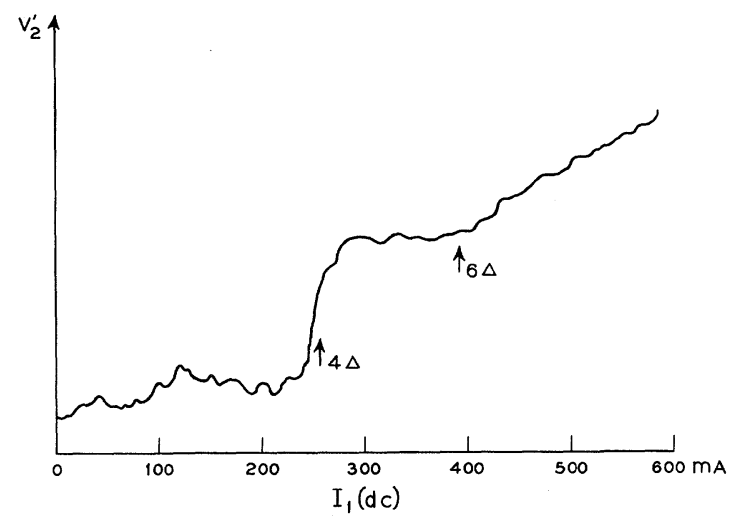

FIG. 3. Derivative of receiver signal of Fig. 2(b) versus generator direct current $I_{1}$. (A fixed 35-mA pulse is superposed on $I_{1}$.) 
es intimately dependent on the voltage applied to the generator. The receiver response cannot be explained by assuming that a heat pulse (black-body radiation) is generated due to a temperature rise associated with the input power to the transmitter.

A simple explanation of our experimental results is obtained if we assume that the receiving diode responds only to phonons of energy $\geqslant 2 \Delta$. Such phonons are absorbed by Cooper pairs with each pair dissociating into two quasiparticles. These quasiparticles occupy states above the gap edge and result therefore in an increase in the tunnel current at the bias voltage $V_{2}<2 \Delta$. Tewordt ${ }^{1}$ calculated that the lifetime of phonons of energy $\gtrsim 2 \Delta$ in a superconductor is $\sim \frac{2}{3}$ the corresponding value in the normal state.

For phonon energy $<2 \Delta$, phonon-assisted tunneling ${ }^{5,6}$ may contribute to the increase of the tunneling current of the receiver. Our results, however, show that this contribution must be negligibly small in comparison with that due to phonon absorption by Cooper pairs, as would be expected theoretically.

If a voltage $V_{1}$ is applied to the generating diode, the tunneling process produces excited quasiparticles of energy $E$ in the range $\Delta \leqslant E$ $\leqslant V_{1}-\Delta$. In general, each excited particle undergoes two relaxation processes. First, there is a relaxation to the top of the energy gap with the emission of a phonon of energy $E-\Delta$ and then a recombination with another excited particle of opposite spin to form a Cooper pair with the emission of a phonon of energy $2 \Delta$. The lifetime $\tau_{s}$ of the relaxation process ${ }^{1}$ is $\infty>\tau_{s}>0.7 \tau_{n}$ for $0 \leqslant(E-\Delta) \leqslant 1.4 \Delta$, where $\tau_{n}$ is the normal state lifetime. The recombination lifetime is probably about two orders of magnitude larger than $\tau_{s}$. We assume that all lifetimes are sufficiently small so that the steady state is achieved within a small fraction of the pulse applied to the generating diode. In the steady state the rate of emission of phonons of energy $2 \Delta$ is approximately equal to that of phonons of energy $E-\Delta$ and each is proportional to $I_{1}$. It is obvious that for $V_{1} \geqslant 4 \Delta$, the excited quasiparticles in the energy range $\Delta$ $\leqslant E \leqslant 3 \Delta$ will emit only one phonon of energy $\geqslant 2 \Delta$; while in the range $3 \Delta \leqslant E \leqslant V_{1}-4 \Delta$, the two phonons emitted are both of energy $\geqslant 2 \Delta$. Thus, if the received signal is due solely to pair dissociation by absorption of phonons of energy $\geqslant 2 \Delta$, the dependence of the receiver signal on $I_{1}$ will exhibit a change in slope similar to that shown in Fig. 2.

The deviation from the straight line which occurs at $V_{1} \geqslant 6 \Delta$ probably indicates the presence of pair dissociation via electron-electron interaction. This is the most effective way a quasiparticle of energy $>5 \Delta$ in the generator or $>3 \Delta$ in the receiver can break up an extra Cooper pair. However, the absence of a sudden change in slope at $V_{1}=6 \Delta$ and the smallness of the deviation from the straight line indicates the predominance of the first-order process even for $V_{1}>6 \Delta$.

In Fig. 2 one observes that the slope of the straight line for $4 \Delta \leqslant V_{1} \leqslant 6 \Delta$ is $\sim 2.5$ times rather than exactly twice that occurring at smaller voltages. Since the relaxation to the top of the gap is definitely associated with phonon emission, the conclusion is reached that the recombination does not occur exclusively via phonon interaction. It is quite possible that about 20 to $40 \%$ of the recombination is via photon emission, or, since photon recombination is rather small theoretically, ${ }^{2,7}$ via some other process involving impurities or surface.

We wish to thank B. I. Miller for several helpful discussions and suggestions. It is a pleasure to acknowledge many fruitful conversations with the following colleages: S. J. Buschsbaum, P. K. Tien, P. W. Anderson, W. L. McMillan, and M. R. Schroeder. The assistance of Mrs. A. A. Pritchard in preparing the samples is also gratefully acknowledged.

\footnotetext{
* Present address: Physikalisches Institut der Universität, Göttingen, West Germany.

${ }^{1}$ L. Tewordt, Phys. Rev. 127, 371 (1962); 128,12 (1962).

${ }^{2}$ D. M. Ginzberg, Phys. Rev. Letters 8 , 204 (1962); J. R. Schrieffer and D. M. Ginzberg, Phys. Rev. Letters $\underline{8}, 207$ (1962).

${ }^{3}$ R. J. von Gutfeld and A. H. Nethercot, Jr., Phys. Rev. Letters 12, 641 (1964); J. M. Andrews, Jr., and M. W. P. Strandberg, Proc. IEEE 54, 523 (1966).

${ }^{4}$ B. T. Bernstein, J. Appl. Phys. 34, 169 (1963).

${ }^{5}$ B. Abeles and Y. Goldstein, Phys. Rev. Letters 14, 595 (1965); E. Lax and Frank L. Vernon, Jr., Phys. Rev. Letters 14, 259 (1965).

${ }^{6}$ L. Kleinman, B. N. Taylor, and E. Burnstein, Rev. Mod. Phys. 36, 208 (1964).

${ }^{7}$ E. Burstein, D. N. Langenberg, and B. N. Taylor, Phys. Rev. Letters $\underline{6}, 92$ (1961).
} 